\title{
Radial metallicity profiles for a large sample of galaxy clusters observed with XMM-Newton
}

\author{
A. Leccardi ${ }^{1,2}$ and S. Molendi ${ }^{2}$ \\ 1 Università degli Studi di Milano, Dip. di Fisica, via Celoria 16, 20133 Milano, Italy \\ 2 INAF - IASF Milano, via Bassini 15, 20133 Milano, Italy \\ e-mail: leccardi@iasf-milano.inaf.ot
}

Received 2 May 2008 / Accepted 4 June 2008

\begin{abstract}
Aims. We measured radial metallicity profiles for a sample of $\approx 50$ hot, intermediate redshift galaxy clusters, selected from the XMM-Newton archive.

Methods. As in our previous paper, we used background modeling rather than background subtraction, and the Cash statistic rather than the $\chi^{2}$. This method requires a careful characterization of all background components. We also performed Montecarlo simulations to assess systematic effects.

Results. The mean metallicity profile shows a peak in the center, and gently declines out to $0.2 R_{180}$. Beyond $0.2 R_{180}$ the metallicity is $\approx 0.2$ solar and, at variance with recently published expectations based on simulations, consistent with being flat. We find no evidence of profile evolution from $z=0.1$ to $z=0.3$. When comparing our mean profile to those obtained by recent works with BeppoSAX and Chandra, we find remarkable agreement over the entire radial range.
\end{abstract}

Key words. X-rays: galaxies: clusters - galaxies: clusters: general - cosmology: observations

\section{Introduction}

Clusters of galaxies are the most massive gravitationally bound systems in the universe. They are permeated by the hot, X-ray emitting intra-cluster medium (ICM), which represents the dominant baryonic component. The mean metallicity of the ICM is found to be roughly half of the solar value (Loewenstein 2004), therefore a substantial fraction of plasma is not of primordial origin. As heavy elements are only produced in stars, the processed material must have been ejected by cluster galaxies into the ICM. In rich clusters the plasma reaches temperatures of several $10^{7} \mathrm{~K}$ and emits X-rays mainly via thermal bremsstrahlung. At such temperatures, most elements are either fully ionized or in a high ionization state. The strong emission lines due to the transitions to the $n=1$ level of the H-like and He-like ions of iron around $6.7 \mathrm{keV}$ (rest frame energy) are the most prominent features in X-ray spectra of hot clusters. In fact, for hot clusters as those belonging to our sample, the measurement of the metallicity is a measurement of the iron abundance.

Spatially resolved analysis of metal abundance in clusters has become possible only recently, first with ASCA and BeppoSAX and then with Chandra and XMM-Newton. These measurements (e.g. De Grandi \& Molendi 2001; De Grandi et al. 2004; Tamura et al. 2004; Baldi et al. 2007) have shown that abundance gradients are common features in clusters of galaxies: most clusters show a peak of metallicity in the center and a gentle decline outward.

In this paper we present radial metallicity profiles from the same sample of hot intermediate redshift clusters analyzed in our previous paper (Leccardi \& Molendi 2008), hereafter Paper I. We used the same novel data analysis technique, which is extensively described in Paper I and summarized in Sect. 3. In Sect. 4.1 we show the mean metallicity profile, in Sect. 4.2 search for an indication of the evolution of the metallicity with redshift, and in Sects. 4.3 and 4.4 compare our mean profile with those obtained from hydrodynamic simulations and from previous observational works. In the Appendix A we describe a systematic effect, which could affect the measurement of the metallicity especially for the case of low metallicity, statistically poor spectra.

Quoted confidence intervals are $68 \%$ for one interesting parameter (i.e. $\Delta C=1$ ), unless otherwise stated. All results are given assuming a $\Lambda \mathrm{CDM}$ cosmology with $\Omega_{\mathrm{m}}=0.3, \Omega_{\Lambda}=0.7$, and $H_{0}=70 \mathrm{~km} \mathrm{~s}^{-1} \mathrm{Mpc}^{-1}$.

\section{The sample}

We selected from the XMM-Newton ${ }^{1}$ archive a sample of hot $(k T>3.3 \mathrm{keV})$, intermediate redshift $(0.1 \lesssim z \lesssim 0.3)$, and high galactic latitude $\left(|b|>20^{\circ}\right)$ clusters of galaxies. We retrieved from the public archive all observations of clusters satisfying the above selection criteria, performed before March 2005 (when the CCD6 of EPIC-MOS1 was switched off ${ }^{2}$ ) and available at the end of May 2007. We excluded from the sample observations that are highly affected by soft proton flares and observations of clusters that show evidence of recent and strong interactions. In Table 3 of Paper I we list the 48 observations that survived our selection criteria and report cluster physical properties. Each observation was performed by using THIN1 or MEDIUM filters, and its total (i.e. MOS1 + MOS2) exposure time is greater than $16 \mathrm{ks}$.

\footnotetext{
1 An ESA science mission with instruments and contributions directly funded by ESA Member States and NASA.

2 http://xmm.esac.esa.int/external/xmm_news/items/ MOS1-CCD6/index . shtml
} 


\section{Data analysis}

In this section we only recall the major steps of our data analysis procedure and refer to Paper I for a detailed description. We stress that in our procedure we used only EPIC-MOS data, because a robust characterization of EPIC-pn background was not possible, mainly due to the small regions of the detector outside the field of view, and to the non-negligible fraction of out-oftime events (see Appendix B in Paper I for further details).

\subsection{Spectra preparation}

Observation data files (ODF) were retrieved from the XMMNewton archive and processed in a standard way with the Science Analysis System (SAS) v6.1.

The soft proton cleaning was performed using a double filtering process, first in a hard $(10-12 \mathrm{keV})$ and then in a soft (2-5 keV) energy range. We filtered the event file according to PATTERN and FLAG criteria, and excluded the "bright" CCDs, i.e. CCD-4 and CCD-5 for MOS1 and CCD-2 and CCD-5 for MOS2 (see Appendix A in Paper I for a discussion). Bright point-like sources were detected, using a procedure based on the SAS task edetect_chain, and excluded from the event file.

The $R_{\mathrm{SB}}$ indicator, i.e. the ratio between surface-brightness calculated inside and outside the field of view (see Eq. (1) in Paper I), allowed us to quantify the amount of the quiescent soft proton (QSP) component survived the double filtering process. Values of $R_{\mathrm{SB}}$ roughly span the range from 1.0 (negligible contamination) to 1.5 (high contamination). Since the observation 0084230401 of Abell 267 is extremely polluted by QSP $\left(R_{\mathrm{SB}}=1.8\right)$, we excluded it from the sample.

The cluster emission is divided in 10 concentric rings (namely $0^{\prime}-0.5^{\prime}, 0.5^{\prime}-1^{\prime}, 1^{\prime}-1.5^{\prime}, 1.5^{\prime}-2^{\prime}, 2^{\prime}-2.75^{\prime}, 2.75^{\prime}-3.5^{\prime}$, $3.5^{\prime}-4.5^{\prime}, 4.5^{\prime}-6^{\prime}, 6^{\prime}-8^{\prime}$, and $10^{\prime}-12^{\prime}$ ) at fixed angular radii, to maintain under control systematics related to the detectors (see the Appendices of Paper I). The most external ring was used to estimate background parameters only. The width of most of the rings is limited by the PSF of the XMM-Newton telescopes. The center of the rings was determined by surface-brightness isocontours at large radii and is usually, but not necessarily, coincident with the X-ray emission peak. We prefer that azimuthal symmetry be preserved at large radii, where we are interested in characterizing profiles, at the expense of central regions. For each instrument (i.e. MOS1 and MOS2) and each ring, we accumulated a spectrum and generated an effective area (ARF). For each observation we generated one redistribution function (RMF) for MOS1 and one for MOS2. We performed a minimal grouping to avoid channels with no counts, as required by the Cash statistic.

\subsection{Spectral analysis}

The spectral analysis followed a substantially new ${ }^{3}$ approach: we used the background modeling, rather than the subtraction, and the Cash statistic rather than the $\chi^{2}$. We fit spectra with an absorbed thermal (WABS*MEKAL in XSPEC v11.3 ${ }^{4}$ ) plus background model in the $0.7-10.0 \mathrm{keV}$ energy band, which represents a good trade off between statistical quality and level of systematics. The details of the background model are reported in the Appendices of Paper I. To model the background, a

\footnotetext{
3 A somewhat similar procedure was already used by Stanford et al. (2001).

4 http://heasarc.nasa.gov/docs/xanadu/xspec/xspec11/ index.html
}

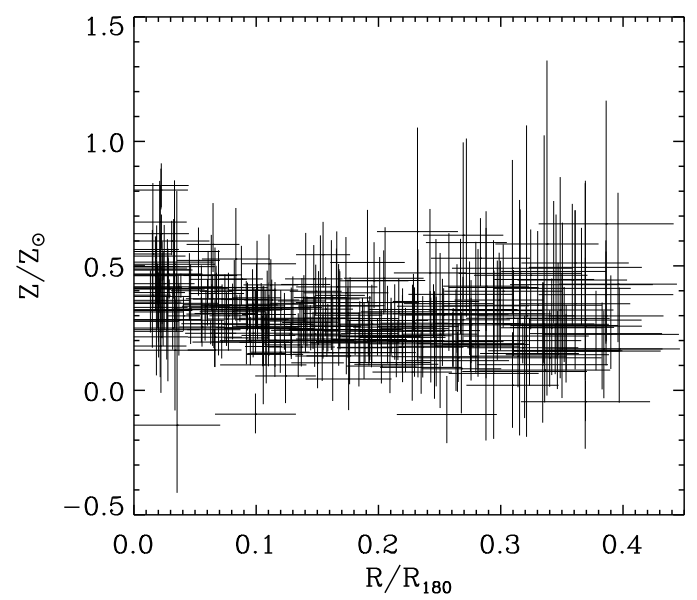

Fig. 1. Radial metallicity profiles for all clusters in our sample. Abundances are expressed in Anders \& Grevesse (1989) solar values and radii in units of $R_{180}$.

careful characterization of all its components is mandatory. Ideally, one would like to estimate background parameters in the same region and at the same time as the source. Since this was not possible, we estimated background parameters in the external $10^{\prime}-12^{\prime}$ ring and rescaled them in the inner rings, by making reasonable assumptions on their spatial distribution tested by analyzing blank-field observations.

We fit spectra in internal rings leaving the temperature and the normalization free to vary; the metallicity was constrained between $\pm 5 Z_{\odot}$ (see the discussion in Appendix A). The redshift was constrained between $\pm 7 \%$ of the optical measurement in the two innermost rings and, in the other rings, was fixed to the average value of the first two rings, by considering independently MOS1 and MOS2 spectra. The main reason for our choice is to allow for EPIC calibration uncertainties, and for possible discrepancies between X-ray and optical derived redshift values. Typical shift values are on the order of $2 \%$. Finally, we produced metallicity profiles for each cluster, by computing the average over the two MOS values.

\section{Metallicity profiles}

\subsection{The mean profile}

In Fig. 1 we show radial metallicity profiles for all clusters in our sample. All our metallicity measurements are relative to the solar values published by Anders \& Grevesse (1989). Even if these have been superseded by more recent values (Grevesse \& Sauval 1998; Asplund et al. 2005), they allow straightforward comparison with most of the literature (De Grandi \& Molendi 2001; Balestra et al. 2007; Baldi et al. 2007). Grevesse \& Sauval (1998) and Asplund et al. (2005) introduced a 0.676 and 0.60 times lower iron solar abundance respectively, while other elements are substantially unchanged. A simple scaling by 0.676 and 0.60 converts measures from the Anders \& Grevesse (1989) iron abundance to the Grevesse \& Sauval (1998) and Asplund et al. (2005) abundances.

The radius is rescaled by $R_{180}$, i.e. the radius encompassing a spherical density contrast of 180 with respect to the critical density. We compute $R_{180}$ from the mean temperature, $k T_{\mathrm{M}}$, and the redshift, $z$, as in Paper I and in Arnaud et al. (2005):

$R_{180}=1780\left(\frac{k T_{\mathrm{M}}}{5 \mathrm{keV}}\right)^{1 / 2} h(z)^{-1} \mathrm{kpc}$, 
Table 1. Mean metallicity profile.

\begin{tabular}{ll}
\hline \hline Ring $^{a}$ & Metallicity \\
\hline $0.00-0.04$ & $0.450 \pm 0.010$ \\
$0.04-0.08$ & $0.352 \pm 0.009$ \\
$0.08-0.12$ & $0.294 \pm 0.010$ \\
$0.12-0.18$ & $0.262 \pm 0.010$ \\
$0.18-0.24$ & $0.230 \pm 0.013$ \\
$0.24-0.32$ & $0.245 \pm 0.017$ \\
$0.32-0.45$ & $0.218 \pm 0.023$ \\
\hline
\end{tabular}

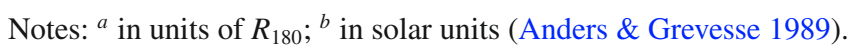

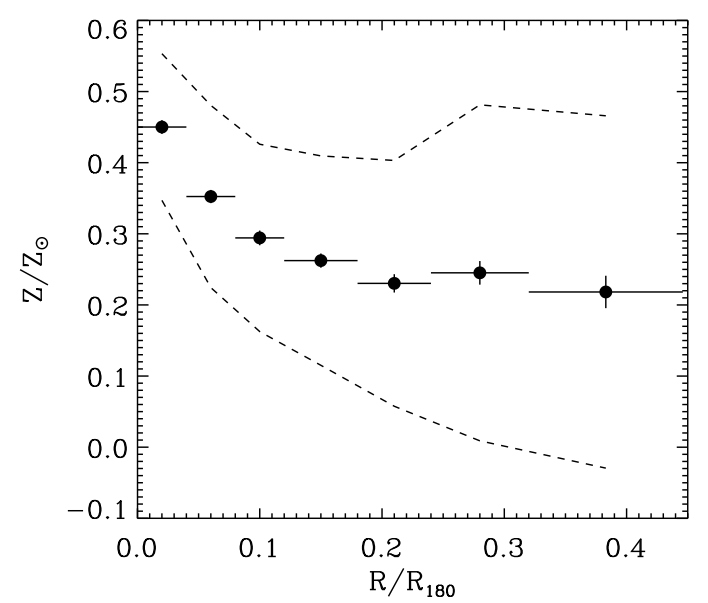

Fig. 2. Mean metallicity profile averaged over all clusters. Abundances are expressed in Anders \& Grevesse (1989) solar values and radii in units of $R_{180}$. The dotted lines show the one-sigma scatter of the values around the average.

where $h(z)=\left(\Omega_{\mathrm{M}}(1+z)^{3}+\Omega_{\Lambda}\right)^{1 / 2} \cdot R_{180}$ is a good approximation to the virial radius in an Einstein-De Sitter universe and has been largely used to rescale cluster radial properties (e.g. De Grandi et al. 2004; Vikhlinin et al. 2005; Pratt et al. 2007; Leccardi \& Molendi 2008). The profiles are limited to $\approx 0.4 R_{180}$ because beyond this radius the source-to-background count rate ratio is too small and the measurements are unreliable (see Sect 3.2.2 in Paper I).

In Table 1 and in Fig. 2 we report the mean metallicity profile binned in units of $R_{180}$; these values have been computed by performing, for each new bin, a weighted average of metallicity values in the original bins which have a non-zero intersection with the new bin. The weight is the product of two components: one is the inverse squared errors, the other depends on the intersection between the original bin and the new one. If the original bin is totally included into the new one, the weight is equal to one. If the original bin has only a partial intersection with the new one, the weight is the fraction of the original bin that belongs to the new one. Possible blurring effects associated either to the original binning in angular units or to the recasting in units of $R_{180}$ should be minimal. As far as the original binning is concerned, we note that the size of central bins (i.e. $30^{\prime \prime}$ ) is comparable to the XMM-Newton PSF. As far as the recasting is concerned, the one we show in Fig. 2 is the result of various trials specifically aimed at reaching the best compromise between resolution and statistical quality; moreover, the size of central bins (i.e. $0.04 R_{180}$ ) corresponds to $\approx 30^{\prime \prime}$ for clusters at $z \approx 0.2$ with a temperature of $7 \mathrm{keV}$.

Balestra et al. (2007) and Maughan et al. (2008) have adopted a procedure that is alternative to ours to estimate mean

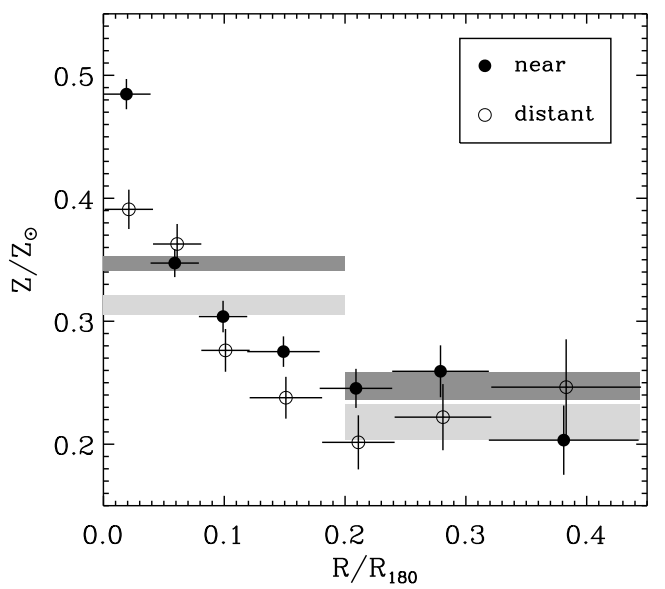

Fig. 3. Mean metallicity profiles for near $(z<0.2$, filled circles) and distant $(z>0.2$, empty circles) clusters. The dark and the light shaded regions indicate the average metallicity within one-sigma uncertainties below and beyond $0.20 R_{180}$ for near and distant clusters, respectively. The radii have been slightly offset in the plot for clarity.

metal abundances for a sample of clusters. These authors performed a simultaneous spectral fit, leaving temperature and normalization free to vary for each object and using a unique metallicity value for all clusters in each redshift bin. We note that when modeling the background, as we do, a joint fit is infeasible, because of the large number of model parameters. Moreover, we are not aware of any detailed work that investigates the impact of systematic errors, possibly affecting individual measurements, on the final result of a joint fit.

The mean metallicity is $0.45 Z_{\odot}$ in the center and decreases out to $\approx 0.2 R_{180}$; beyond this radius the profile is consistent with being flat, a fit with a constant for $R>0.2 R_{180}$ gives $Z=0.23 \pm 0.01 Z_{\odot}$. The profiles show a large scatter, which is mostly of statistical origin. In the central regions $\left(R<0.2 R_{180}\right)$ we find an intrinsic scatter of $22 \pm 2 \%$ related to the presence of cool core clusters; in the outer regions $\left(R>0.2 R_{180}\right)$ the intrinsic scatter is only $14 \pm 8 \%$ (i.e. $\approx 0.03 Z_{\odot}$ ), the same order of magnitude as our systematics (see Appendix A). Past works (e.g. De Grandi \& Molendi 2001) have shown that the abundance profiles of cool core and non cool core clusters (see Sect. 4 in Paper I) differ in the central regions. We found qualitatively similar results, but we choose to extensively address this important issue in a forthcoming paper (Leccardi et al., in prep.), where we also compare our results with those obtained from a local sample.

\subsection{Redshift evolution}

We divided clusters in our sample into two groups to investigate a possible profile evolution with redshift: near (distant) clusters are characterized by a redshift lower (greater) than 0.2.

In Fig. 3 we show the mean metallicity profiles for the two groups. The single points are consistent within one- or twosigma, except for the core region where the spatial resolution plays an important role. In our procedure we fixed the size of the central ring to $30^{\prime \prime}$, which corresponds to $\approx 0.03 R_{180}$ for nearest $(z \approx 0.1)$ and to $\approx 0.07 R_{180}$ for most distant $(z \approx 0.3)$ objects; thus, the metallicity peak for distant clusters is blurred over a larger region. The three-sigma discrepancy in the region within $0.2 R_{180}$ (see shaded regions in Fig. 3 ) is most likely due to a different fraction of cool core and non cool core clusters within the two subsamples (Leccardi et al., in prep.). 
In the outer regions, if the metallicity is allowed to vary between 0 and $5 Z_{\odot}$ as commonly done, we measure a discrepancy between two profiles of $0.061 \pm 0.018 Z_{\odot}$ with a more than three-sigma significance (see Appendix A). Conversely, if the metallicity is allowed to vary between $\pm 5 Z_{\odot}$ as in our data analysis procedure (see Sect. 3.2), near and distant clusters have a mean metallicity of $0.248 \pm 0.011 Z_{\odot}$ and $0.219 \pm 0.015 Z_{\odot}$, respectively. For this case, the discrepancy (i.e. $\approx 1.5$ sigma, see shaded regions in Fig. 3) is consistent with a purely statistical fluctuation, and is the same order of magnitude as our systematics. Summarizing, we find no evidence of metallicity profile evolution from $z=0.1$ to $z=0.3$. We stress that the systematic effect described in the Appendix A, which affects in particular low metallicity, statistically poor spectra, if unaccounted for, could cause a false detection of the metallicity evolution.

Two recent works (Balestra et al. 2007; Maughan et al. 2008) have investigated the evolution in the iron content of the ICM, by analyzing data from the Chandra archive. As mentioned in Sect. 4.1, these authors performed a simultaneous spectral fit, leaving temperature and normalization free to vary for each object and using a unique metallicity value for all clusters in each redshift bin. Unfortunately, we cannot compare our results with those obtained by Balestra et al. (2007), because they considered different regions from cluster to cluster. Instead, Maughan et al. (2008) analyzed the region within $R_{500}\left(\approx 0.6 R_{180}\right)$ with and without the core region (i.e. $0.15 R_{500}$ ) and obtained respectively $Z \approx 0.4 Z_{\odot}$ and $Z \approx 0.35 Z_{\odot}$ between $z=0.1$ and $z=0.3$. When analyzing roughly the same regions for our clusters, we obtain $Z=0.32 Z_{\odot}$ and $Z=0.26 Z_{\odot}$ respectively, with negligible uncertainties; i.e., a significantly lower $(\approx 20 \%)$ mean metallicity. A possible explanation for this discrepancy may be related to different weights on the averaging procedure: we averaged weighting over the inverse squared errors, while Maughan et al. (2008) measured metal abundances from individual spectra extracted from the entire region of interest.

\subsection{Comparison with hydrodynamic simulations}

We compare our (hereafter LM08) mean metallicity profile with the one (hereafter F08) derived from hydrodynamic simulations of four relaxed clusters by Fabjan et al. (2008).

The simulations are performed using the hydrodynamical TREE-SPH code GADGET-2 (Springel 2005) with the implementation of chemical enrichment by Tornatore et al. (2007). The authors used the emission-weighted definition of metallicity, with emissivity of each gas particle computed in the $0.5-10.0 \mathrm{keV}$ energy band. In principle, for a comparison with observational data, one should extract synthetic spectra from the simulated clusters and then measure the metallicity by fitting these spectra with a single-temperature and single-metallicity plasma model (e.g. the MEKAL model in XSPEC). A recent work presented by Rasia et al. (2008) showed that, at least for Iron, the emission-weighted estimator of the metallicity gives results quite close (within about $10 \%$ ) to those obtained from the spectral-fitting analysis.

In Fig. 4 we compare LM08 and F08 profiles. We note differences at both small and large radii. In the central region, the F08 profile is much more peaked; however, this difference likely results from two factors: namely that Fabjan et al. (2008) analyzed only relaxed clusters and that our data are limited by the XMM-Newton PSF. At large radii, while the F08 profile shows a constant decrease, ours is consistent with being flat beyond $\approx 0.2 R_{180}$. This discrepancy could be due to issues related to observations, simulations, or both. More precisely, observations

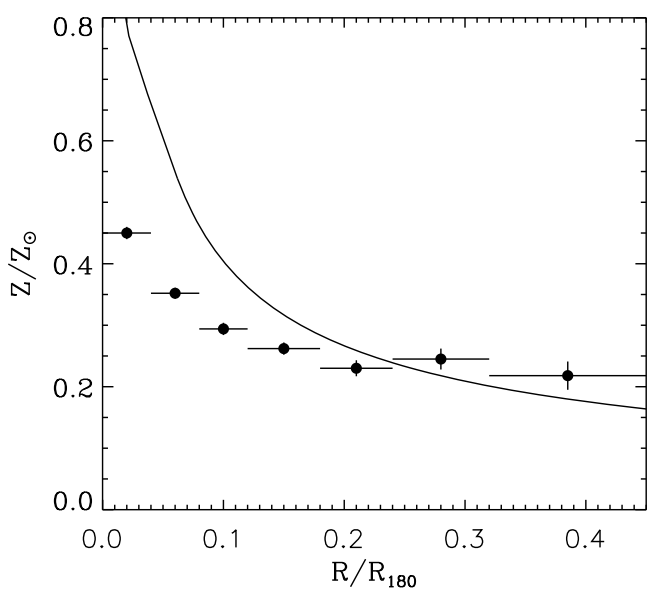

Fig. 4. Comparison between our observed mean profile (circles) and the one derived from hydrodynamic simulations (solid line) by Fabjan et al. (2008). Abundances are expressed in Anders \& Grevesse (1989) solar values and radii in units of $R_{180}$.

could be affected by unknown systematic effects, and simulations could underestimate the metallicity at large radii by underestimating possible convective motions in the ICM, which might be responsible of mixing metals.

\subsection{Comparison with previous works}

We compare our results with those obtained by De Grandi et al. (2004) and Baldi et al. (2007). De Grandi et al. (hereafter DM04) have analyzed a sample of 21 hot $(k T \gtrsim 3.5 \mathrm{keV})$, nearby $(z \lesssim 0.1)$ galaxy clusters observed with BeppoSAX. Baldi et al. (hereafter BA07) have analyzed 12 very hot $(k T \gtrsim 6 \mathrm{keV})$, intermediate redshift $(0.1 \lesssim z \lesssim 0.3)$ clusters observed with Chandra.

Comparing results obtained from different works is not trivial; indeed, cluster physical properties, instrumental characteristics, and data analysis procedures may differ. Moreover, each author uses his own recipe to derive a scale radius. We have rescaled DM04 and BA07 profiles by using the standard cosmology (see Sect. 1) and deriving the scale radius, $R_{180}$, as explained in Sect. 4.1; the aim is to reduce all inhomogeneities as much as possible. We also converted DM04 abundances from Grevesse \& Sauval (1998) to Anders \& Grevesse (1989) solar values.

In Fig. 5 we compare LM08, DM04, and BA07 mean metallicity profiles. Even if cluster samples cover a different redshift range and the instruments (i.e. XMM-Newton, BeppoSAX, and Chandra) present different characteristics, the mean profiles are remarkably similar over the entire radial range.

\section{Summary and conclusions}

We analyzed a sample of $\approx 50$ hot, intermediate redshift galaxy clusters to measure their radial properties, in this paper we focused on metallicity profiles. Our main results are summarized as follows.

- The mean metallicity is $0.45 Z_{\odot}$ in the center and decreases out to $\approx 0.2 R_{180}$; beyond $0.2 R_{180}$ the metallicity is consistent with being flat at $0.23 \pm 0.01 Z_{\odot}$.

- The profiles show a large scatter, which is mostly of statistical origin. In the central regions the scatter (i.e. $22 \pm 2 \%$ ) is also related to the presence of cool core clusters, in the outer regions it (i.e. $14 \pm 8 \%$ ) is comparable to systematics. 


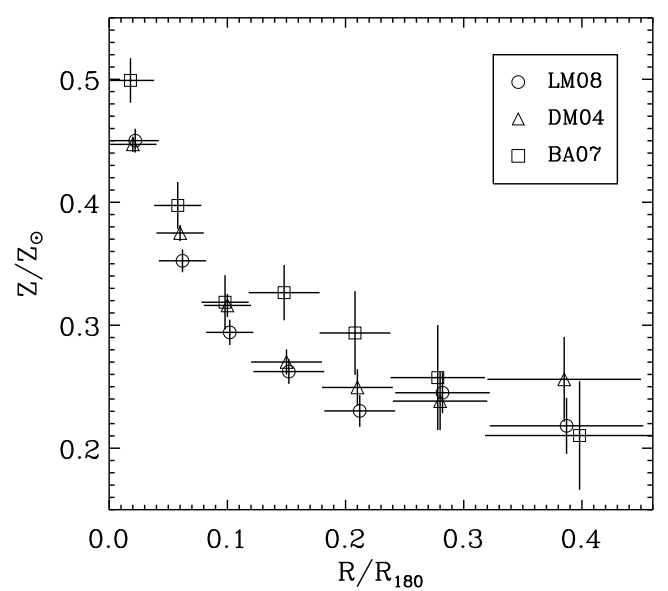

Fig. 5. Mean metallicity profiles obtained from this work (LM08, circles), by De Grandi et al. (2004) (DM04, triangle), and Baldi et al. (2007) (BA07, squares). Abundances are expressed in Anders \& Grevesse (1989) solar values and radii in units of $R_{180}$. The radii have been slightly offset in the plot for clarity.

- There is no evidence of profile evolution from $z=0.1$ to $z=0.3$.

- We find a mean metallicity $\approx 20 \%$ lower than found by Maughan et al. (2008) using Chandra, when analyzing roughly the same regions for clusters in the same redshift range.

- We point out the existence of a systematic effect, affecting in particular distant clusters, which, if unaccounted for, could cause a false detection of evolution.

- When comparing our mean profile to the one derived from hydrodynamic simulations by Fabjan et al. (2008), we find differences at small and large radii. In particular, while the profile obtained by Fabjan et al. (2008) shows a constant decrease, ours is consistent with being flat beyond $\approx 0.2 R_{180}$.

- When comparing our mean profile to those obtained by recent works with BeppoSAX (De Grandi et al. 2004) and Chandra (Baldi et al. 2007), we find remarkable agreement over the entire radial range.

Our results have been obtained using the same novel data analysis technique as described in Paper I; i.e., the background modeling rather than the background subtraction, and the Cash statistic rather than the $\chi^{2}$.

Acknowledgements. We acknowledge the financial contribution from contracts ASI-INAF I/023/05/0 and I/088/06/0. We thank A. Baldi for kindly providing his data.

\section{Appendix A: Discussion of systematics}

We made use of montecarlo simulations to test the reliability of our metallicity measurements. The simulation procedure is similar to that described for the source-only case in Sect. 2 of Leccardi \& Molendi (2007). We considered a thermal (MEKAL in XSPEC) spectrum only, without a background. Input parameters are $6 \mathrm{keV}$ temperature, $0.25 Z_{\odot}$ metallicity, 0.2 redshift, and $9 \times 10^{-4}$ (in XSPEC units) normalization. We used the Abell 1689 EPIC-MOS1 observation as a guideline, for producing RMF and ARF, and for choosing typical input model parameters. We considered the $3.5^{\prime}-4.5^{\prime}$ ring only. For each channel, we perturbed the number of counts with a Poisson distribution centered on the expected value, and repeated this procedure 1000 times to obtain 1000 spectra, which simulate 1000 independent measurements of the source. We fit simulated spectra

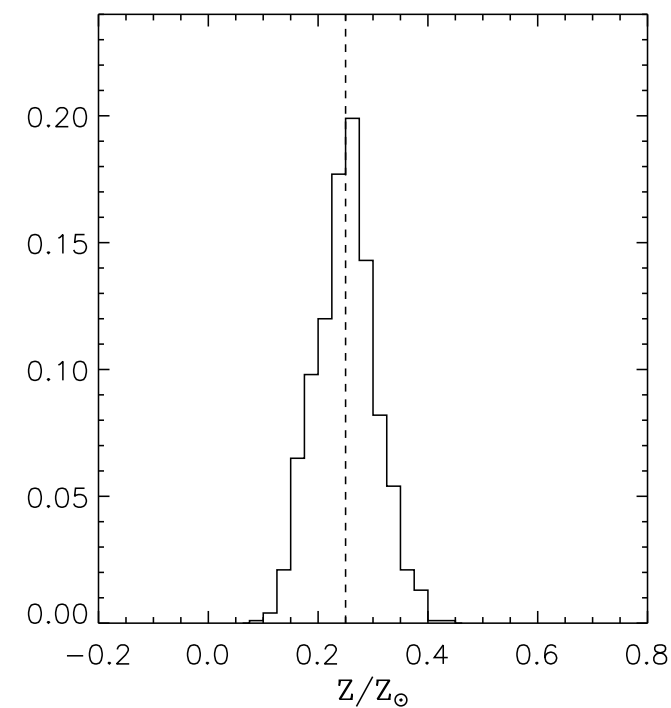

Fig. A.1. Frequency distribution for metallicity (expressed in solar units) best fit values for a $100 \mathrm{ks}$ exposure time. The dashed line indicates the input value, i.e. $0.25 Z_{\odot}$.

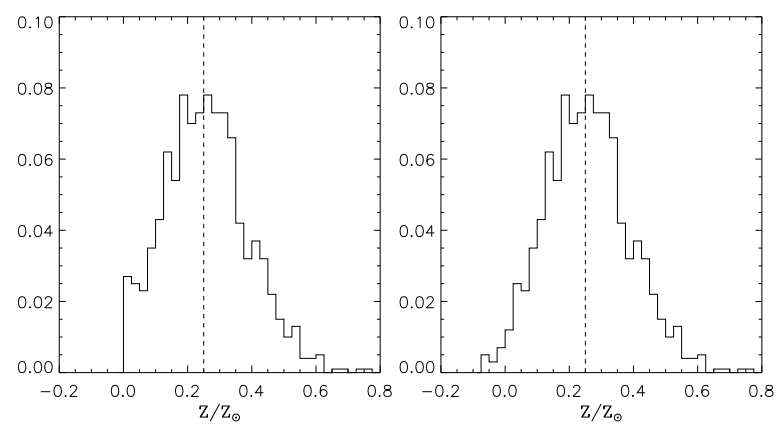

Fig. A.2. Frequency distribution for metallicity (expressed in solar units) best fit values for a $20 \mathrm{ks}$ exposure time. The dashed lines indicate the input value, i.e. $0.25 Z_{\odot}$. In the left panel, the metallicity is forced to be positive; in the right one, it can assume negative values too. The two histograms only differ around zero.

with a MEKAL model in the $0.7-10.0 \mathrm{keV}$ energy band using the Cash statistic. Temperature, metallicity, redshift, and normalization were allowed to vary within their XSPEC standard ranges. Here we focused on the metallicity, for which the standard allowed range is between 0 and $10^{3}$ in solar units. For each measurement, we determined the best fit value and the one-sigma uncertainties.

In Fig. A.1 we report the frequency distribution for metallicity best fit values for a $100 \mathrm{ks}$ exposure time (i.e. for spectra with high statistical quality); as expected, the curve is very similar to a narrow Gaussian peaked around the input value (i.e. $0.25 Z_{\odot}$ ). The mean, the median, and the weighted (over one-sigma uncertainties) average are all close to $0.25 Z_{\odot}$, namely the weighted average is $0.246 \pm 0.002 Z_{\odot}$.

In the left panel of Fig. A. 2 we report the same histogram for a $20 \mathrm{ks}$ exposure time (i.e. for spectra with standard statistical quality). The curve is peaked on the input value, but the variance, which mainly depends on the number of counts around the energy of the emission line, is higher. Since the metallicity is forced to be positive, the curve is truncated and a dozen points pile up near the boundary (i.e. zero). In this case, the mean and the median are close to $0.25 Z_{\odot}$, instead the weighted average is $0.193 \pm 0.003 Z_{\odot}$, i.e. $\approx 25 \%$ lower than the input value. Part of this discrepancy is due to a boundary effect. For 
measurements characterized by a negative fluctuation of the metallicity, the minimum of the $\chi^{2}$ curve lies outside the allowed range. For these cases, the best fit values are forced to be above zero and the uncertainties have unreliable small values; therefore, when computing a weighted average, these measurements have the highest statistical weight, and the net effect is an underestimate of the real metallicity. When fitting exactly the same spectra, allowing the metallicity to be negative, we obtain the histogram reported in the right panel of Fig. A.2. For this case, the tails of the curve are more symmetric, all measurements have roughly the same uncertainty, as expected, and the weighted average is $0.229 \pm 0.004 Z_{\odot}$, i.e. $\approx 8 \%$ lower than the input value. This simple solution allowed us to correct for most of the underestimate; however, a small (i.e. $\approx 0.02 Z_{\odot}$ ) systematic still affects our measurements, especially in the outer regions. We also performed more realistic simulations, by introducing a background, and obtained substantially similar results.

Although allowing observables to assume unphysical values is against common sense, there are measurement procedures that can yield unphysical values. Deciding to accept only physical values and reject others will clearly result in a bias. This is a general issue, which does not pertain to astrophysics alone. An interesting example we found concerns the analysis of data from the Collider Detector at Fermilab ${ }^{5}$. A statistical committee specifically appointed to provide guidelines for the analysis of the Collider Detector data recommends a treatment similar to the one we propose here, i.e. unphysical values can be used in statistical procedures.

The boundary effect we have just pointed out should play an important role when comparing subsamples characterized by a different statistical quality of the data. For example, in Sect. 4.2 we compare the metallicity obtained for near and distant clusters. In our sample, near cluster spectra usually have a better statistical quality for various reasons (e.g. longer observations, cosmological dimming effect). If the metallicity is allowed to vary between 0 and $5 Z_{\odot}$, the mean metallicity beyond $0.20 R_{180}$ is $0.249 \pm 0.011 Z_{\odot}$ and $0.188 \pm 0.014 Z_{\odot}$, for near and distant clusters respectively. The measured discrepancy of $0.061 \pm 0.018 Z_{\odot}$ has a significance of more than three-sigma. Conversely, if the metallicity is allowed to vary between $\pm 5 Z_{\odot}$ (see Sect. 4.2), the discrepancy of $0.029 \pm 0.019 Z_{\odot}$ is consistent with a purely statistical fluctuation.

We then warn X-ray astronomers about the existence of this kind of systematics, which could affect the measurement of the metallicity, especially for the case of low metallicity, statistically poor spectra.

\section{References}

Anders, E., \& Grevesse, N. 1989, Geochim. Cosmochim. Acta, 53, 197 Arnaud, M., Pointecouteau, E., \& Pratt, G. W. 2005, A\&A, 441, 893

Asplund, M., Grevesse, N., \& Sauval, A. J. 2005, in Cosmic Abundances as Records of Stellar Evolution and Nucleosynthesis, ed. T. G. Barnes, III, \& F. N. Bash, ASP Conf. Ser., 336, 25

Baldi, A., Ettori, S., Mazzotta, P., Tozzi, P., \& Borgani, S. 2007, ApJ, 666, 835

Balestra, I., Tozzi, P., Ettori, S., et al. 2007, A\&A, 462, 429

De Grandi, S., \& Molendi, S. 2001, ApJ, 551, 153

De Grandi, S., Ettori, S., Longhetti, M., \& Molendi, S. 2004, A\&A, 419, 7

Fabjan, D., Tornatore, L., Borgani, S., Saro, A., \& Dolag, K. 2008, MNRAS, 386,1265

Grevesse, N., \& Sauval, A. J. 1998, Space Sci. Rev., 85, 161

Leccardi, A., \& Molendi, S. 2007, A\&A, 472, 21

Leccardi, A., \& Molendi, S. 2008, A\&A, 486, 359

Loewenstein, M. 2004, in Origin and Evolution of the Elements, ed. A. McWilliam, \& M. Rauch, 422

Maughan, B. J., Jones, C., Forman, W., \& Van Speybroeck, L. 2008, ApJS, 174, 117

Pratt, G. W., Böhringer, H., Croston, J. H., et al. 2007, A\&A, 461, 71

Rasia, E., Mazzotta, P., Bourdin, H., et al. 2008, ApJ, 674, 728

Springel, V. 2005, MNRAS, 364, 1105

Stanford, S. A., Holden, B., Rosati, P., et al. 2001, ApJ, 552, 504

Tamura, T., Kaastra, J. S., den Herder, J. W. A., Bleeker, J. A. M., \& Peterson, J. R. 2004, A\&A, 420, 135

Tornatore, L., Borgani, S., Dolag, K., \& Matteucci, F. 2007, MNRAS, 382, 1050

Vikhlinin, A., Markevitch, M., Murray, S. S., et al. 2005, ApJ, 628, 655

\footnotetext{
${ }^{5}$ http://www-cdf.fnal.gov/physics/statistics/ statistics_faq.html\#ssel1
} 